\section{Efecto del 27- hidroxicolesterol en la acción antiplaquetaria de estradiol en mujeres postmenopáusicas}

\author{
GLADYS ROCHA ${ }^{a}$, WALTER SIERRALTA $^{\mathrm{b}}$, LUIS VALLADARES $^{\mathrm{c}}$
}

\section{7- Hydroxycholesterol reverses estradiol induced inhibition of platelet aggregation in postmenopausal women}

Background: The decline of estrogen levels increases cardiovascular risk in women. Platelets express estrogen receptors and 17ß-estradiol- (E2) can produce a protective effect on thrombus formation. The hydroxylation of cholesterol generates several sterols and 27-hydroxycholesterol (27HC) predominates in circulation. Aim: To evaluate the effect of $27 \mathrm{HC}$ as an endogenous antagonist of the anti-aggregating properties of E2 in platelets of postmenopausal women. Material and Methods: Platelet function of postmenopausal women was evaluated ex-vivo. Platelets pre-incubated with $27 \mathrm{HC}$ in the presence or absence of E2, were stimulated with collagen. Aggregation was evaluated using turbidimetry using a Chrono-log aggregometer. Results: Collagen-stimulated platelet aggregation was significantly inhibited by E2. The inhibitory effect of E2 on collagen-stimulated platelet aggregation was significantly reversed in the presence of 27HC. Conclusions: The suppressive effect of E2 on platelet aggregation is inhibited by $27 \mathrm{HC}$, which could contribute to increase cardiovascular risk in postmenopausal women.

(Rev Med Chile 2016; 144: 1377-1381)

Key words: Estradiol; 27-hydroxycholesterol; Platelet Aggregation; Postmenopause.

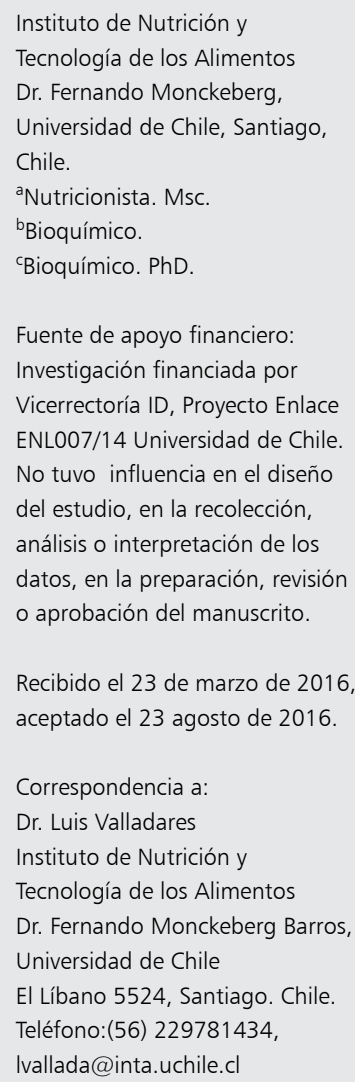

Recibido el 23 de marzo de 2016 aceptado el 23 agosto de 2016 .

Correspondencia a:

Dr. Luis Valladares

Instituto de Nutrición y

Tecnología de los Alimentos

Dr. Fernando Monckeberg Barros,

Universidad de Chile

El Líbano 5524, Santiago. Chile. Teléfono:(56) 229781434,

Ivallada@inta.uchile.cl

E studios epidemiológicos han mostrado que las mujeres pre-menopáusicas están protegidas frente a las enfermedades cardiovasculares (ECV) si las comparamos con los hombres de similar edad. Después de la menopausia, el riesgo cardiovascular aumenta progresivamente; entre los factores que contribuyen al desarrollo de eventos cardiovasculares, se ha descrito la caída de los niveles de estrógenos, especialmente estradiol-17 $\beta$ (E2) ${ }^{1}$. Estos efectos estarían relacionados a la amplia expresión de receptores de estrógenos (ERs) en diversas poblaciones celulares del organismo, lo que probablemente refleja la participación de un gran número de moléculas que pueden interactuar con los ERs ${ }^{2}$. Hay dos iso- formas genéticamente distintas de ERs $(\alpha$ y $\beta)$ que difieren en parámetros de expresión, afinidad de unión al ligando y actividad biológica. Aunque la respuesta biológica específica puede ser atribuida a una acción agonista de ER $\alpha$ y ER $\beta$, se postula que en las células que expresan ambos receptores, el ER $\beta$ parece amortiguar la actividad transcripcional de ER $\alpha$. Así, la respuesta farmacológica de las células blanco a los estrógenos y anti-estrógenos representaría un efecto coordinado sobre ambos receptores ${ }^{3}$.

A mayor edad, la mujer también tiene mayores probabilidades de tener elevados factores de riesgo, específicamente el colesterol. Al respecto, se ha planteado que el colesterol, a través de metabolitos 
hidroxilados, puede bloquear los efectos benéficos de los estrógenos en el sistema cardiovascular. La hidroxilación del colesterol genera diversos oxiesteroles, donde el 27-hidroxicolesterol (27HC) es el de mayor preponderancia en la circulación ${ }^{4}$. El 27HC es un ligando de ERs y tiene la propiedad de inhibir tanto las acciones genómicas como no genómicas de E2 en el sistema cardiovascular (SCV)

Aunque los estrógenos tienen efectos en el ateroma arterial y también en el riesgo de trombosis venosa, sus efectos en la función plaquetaria son poco claros. Los estudios de agregación basados en el tratamiento in vitro de las plaquetas con E2 no siempre ha producido resultados consistentes con la actividad antitrombótica de E2 observada in vivo. Entre las variables que podrían introducir sesgo en los ensayos in vitro de la agregación plaquetaria femenina está el efecto de los antagonistas endógenos de los ERs ${ }^{6}$.

El propósito de este estudio, ex-vivo, fue analizar el impacto de 27HC en la homeostasis de la mujer posterior a la cesación de estrógenos por el ovario. Se espera que el $27 \mathrm{HC}$ anule el efecto inhibitorio de estradiol en la agregación plaquetaria. Para esto estudiamos el efecto del oxisterol sobre la actividad antiagregante de E2 en plaquetas de mujeres postmenopáusicas.

\section{Materiales y Métodos}

El protocolo del estudio fue revisado y aprobado por el Comité de Ética del Instituto de Nutrición y Tecnología de los Alimentos, Universidad de Chile.

El colágeno (tipo 1) fue obtenido de Chrono-Log (Havertown, PA, EUA), 27-hydroxicolesterol fue obtenido de Cayman (Ann Arbor, MI, EUA). Todos los otros reactivos químicos provenían de Sigma-Aldrich (Atlanta, GA, EUA).

\section{Sujetos}

Se trabajó con una muestra por conveniencia equivalente a once mujeres, edad entre 50 y 73 años y con un IMC $\leq 30$, con consentimiento informado de acuerdo a la Declaración de Helsinki. Para ser incorporadas, las mujeres tenían al menos 6 meses de menopausia, niveles de FSH sobre 20 UI/L, no fumadoras, sin ningún tipo de tratamiento hormonal, sin consumir habitualmente productos derivados de la soja o suplementos dietarios derivados de hierbas. Criterios de exclusión fueron: sin antecedentes personales preexistente de diabetes, hipertensión o dislipidemia.

\section{Preparación de plaquetas y ensayo de agregación}

Las plaquetas fueron preparadas de acuerdo al método descrito previamente ${ }^{7}$. En forma breve, la sangre fue extraída por punción venosa en citrato de sodio (9:1) y centrifugada a $200 \times$ g por $15 \mathrm{~min}$ at $25^{\circ} \mathrm{C}$ para obtener plasma rico en plaquetas (PRP). Este fue eluído en columnas de gel sepharosa-2B con solución de Tyrode-libre de calcio, $\mathrm{pH} 7,4$, con una velocidad de flujo de $1 \mathrm{ml} / \mathrm{min}$. La concentración final de plaquetas fue ajustada a $\sim 2 \times 10^{8} / \mathrm{ml}$. La suspensión de plaquetas $(0,25$ $\mathrm{ml})$ fue pre-incubada con $27 \mathrm{HC}(5$ y $10 \mu \mathrm{M}) \mathrm{y} / \mathrm{o}$ $\mathrm{E} 2,(0,1,1$, y $5 \mu \mathrm{M})$ a 15 y $5 \mathrm{~min}$, respectivamente, antes de la adición de $1 \mu \mathrm{g} / \mathrm{ml}$ de colágeno.

La agregación plaquetaria fue determinada por turbimetría, usando un lumi-agregómetro (Chrono-log). El porcentaje de transmitancia óptica de las plaquetas aisladas fue considerado como $0 \%$ y el del Medio de Tyrode como 100\%. La agregación plaquetaria inducida por colágeno fluctuó entre 85 y $90 \%$ siendo monitorizada durante 6 min. Para los cálculos de acción inhibitoria de los compuestos, el valor de agregación plaquetaria inducida por colágeno en cada experimento fue considerado como $100 \%$.

\section{Análisis estadístico}

Se usó el test no paramétrico de Kruskal Wallis para evaluar si hay diferencia entre 3 o más grupos y el test de Mann-Whitney para identificar diferencia entre pares. En todos los experimentos, el grupo control fue normalizado a $100 \%$. Los resultados se expresan como media y desviación estándar. Un valor p menor a 0,05 fue considerado como significativo. El análisis de datos se realizó con el software Stata E12.

\section{Resultados}

\section{Efecto E2 en la agregación plaquetaria estimulada por colágeno}

Nosotros examinamos la capacidad inhibitoria de E2 en la agregación plaquetaria estimulada por colágeno. Como se observa en la Figura 1, cuando las plaquetas fueron pre-incubadas con E2 $(0,1,1$ y $5 \mu \mathrm{M})$, la agregación estimulada por colágeno $(1 \mu \mathrm{g} /$ ml) fue drásticamente inhibida a $28 \%, 19 \%$ y $11 \%$, respectivamente, comparada con el control (100\%). 


\section{Efecto del 27HC sobre la acción antiplaquetaria} de estradiol

El efecto de 27HC sobre la acción inhibitoria de E2 en la agregación plaquetaria estimulada por colágeno se presenta en la Figura 2. Cuando las plaquetas de mujeres postmenopáusica fueron pre-incubadas con $5 \mu \mathrm{M}$ de $27 \mathrm{HC}$, la acción inhibitoria de $5 \mu \mathrm{M}$ E2 en la agregación plaquetaria inducida por $1 \mu \mathrm{g} / \mathrm{ml}$ de colágeno $(100 \%)$ fue antagonizada significativamente. En presencia de

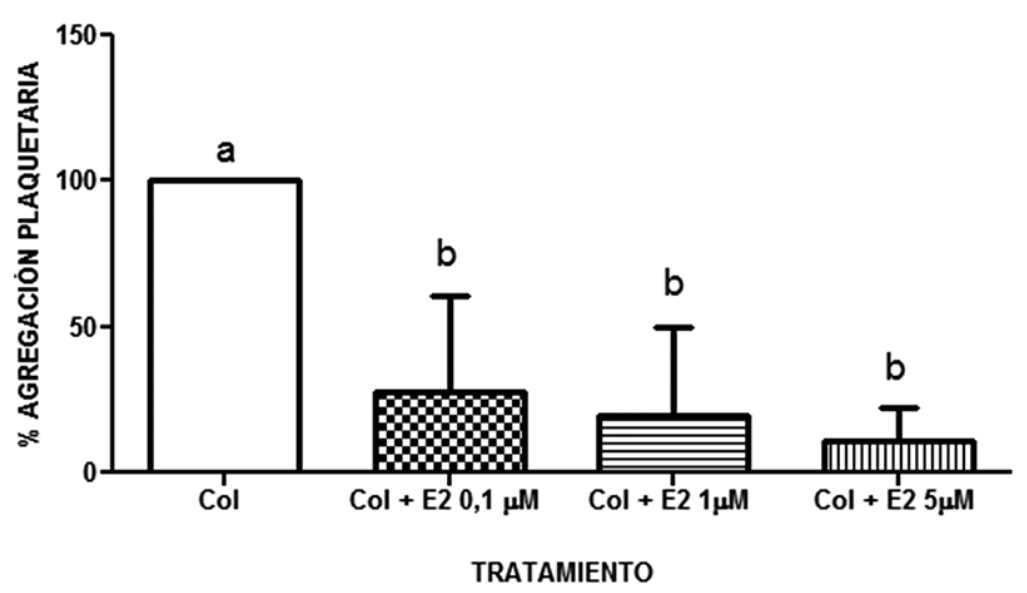

Figura 1. Efecto de estradiol-17 $\beta$ (E2) en la agregación plaquetaria estimulada por colágeno. Las plaquetas lavadas (2 x 108/ $\mathrm{ml}$ ) fueron preincubadas por $5 \mathrm{~min}$, con E2 $(0,1,1$ y $5 \mu \mathrm{M})$ o solvente seguido por la adición de colágeno $(1 \mu \mathrm{g} / \mathrm{ml})$ para inducir la agregación de las plaquetas. Las barras representan media \pm DS de $n=5-11$ experimentos independientes. Los resultados se presentan como porcentaje normalizado a la agregación de plaquetas control que son tratadas con colágeno (100\%). Test de Kruskal-Wallis para diferencia entre los grupos $(p=0,0001)$ y prueba de Mann-Whitney para identificar diferencias respecto al grupo control: Col vs E 0,1 $\mu \mathrm{M}(\mathrm{p}=0,0001)$, Col vs $\mathrm{E} 1 \mu \mathrm{M}(\mathrm{p}=0,0001)$ y Col vs $\mathrm{E} 5 \mu \mathrm{M}(\mathrm{p}=0,0000)$.

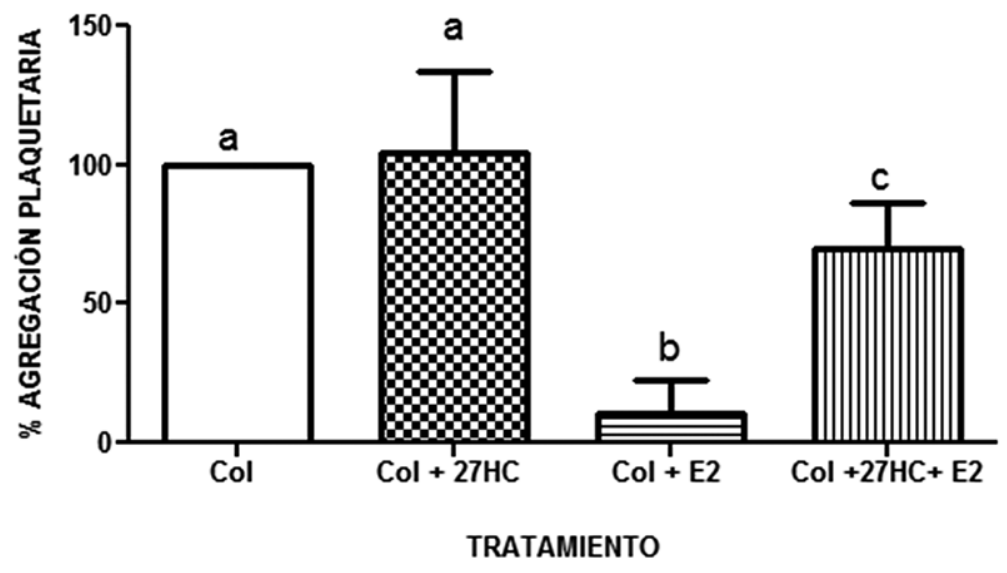

Figura 2. Efecto del 27 hidroxicolesterol $(27 \mathrm{HC})$ sobre la acción antiplaquetaria de estradiol-17 $\beta$ (E2). Las plaquetas lavadas ( $2 \times 108 / \mathrm{ml})$ fueron preincubadas por 15 min con $27 \mathrm{HC}(5 \mu \mathrm{M})$ y 5 min con E2 $(5 \mu \mathrm{M})$ o solvente seguido por la adición de colágeno $(1 \mu \mathrm{g} / \mathrm{ml})$ para inducir la agregación de las plaquetas. Las barras representan media $\pm \mathrm{DS}$ de $\mathrm{n}=9-11$ experimentos independientes. Los resultados se presentan como porcentaje normalizado a la agregación de plaquetas control que son tratadas con colágeno (100\%). Test de Kruskal-Wallis para diferencia entre los grupos $(p=0,0001)$ y prueba de Mann-Whitney para identificar diferencias entre dos grupos: Col vs $27 \mathrm{HC}(p=0.1145)$, Col vs $E(p=0.0000)$, E vs $E+27(p=0,0002)$ y Col vs $27+E(p=0.0000)$. 
$5 \mu \mathrm{M}$ de E2, la agregación plaquetaria estimulada por $1 \mu \mathrm{g} / \mathrm{ml}$ de colágeno fue aproximadamente $11 \%$. Sin embargo, la pre-incubación de las plaquetas con $5 \mu \mathrm{M}$ de $27 \mathrm{HC}$ revirtió significativamente $(\mathrm{p}=0,0002)$ el efecto inhibitorio de $5 \mu \mathrm{M}$ de E2 en la agregación plaquetaria estimulada por 1 $\mathrm{ug} / \mathrm{ml}$ de colágeno $(70 \pm 16,5)$. En la misma Figura 2 se observa que las plaquetas pre-incubadas con $27 \mathrm{HC}$ no afectó significativamente $(\mathrm{p}>0,05)$ la agregación de plaquetas estimulada por $1 \mu \mathrm{g} / \mathrm{ml}$ de colágeno $(104,6+28,7)$ versus $100 \%$ del control.

\section{Discusión}

Después de la menopausia, el riesgo cardiovascular aumenta progresivamente y entre los factores que contribuyen al desarrollo de estas patologías se ha descrito la caída de los niveles de estrógenos, especialmente de E2 ${ }^{1}$. El objetivo de este estudio, ex-vivo, fue estudiar el impacto de los oxiesteroles en la coagulación de la mujer postmenopáusica. Los resultados confirman el dramático efecto que ejerce E2 sobre la agregación plaquetaria ${ }^{6} \mathrm{y}$ demuestran que el $27 \mathrm{HC}$ es un potente inhibidor del efecto antiagregante de estradiol en plaquetas estimuladas por colágeno en mujeres postmenopáusicas. La acción de E2 fue dependiente de la dosis y el mayor efecto antiplaquetario se observó a una concentración de $5 \mu \mathrm{M}$ de E2. Lo anterior confirma los resultados de $\mathrm{Wu}$ et $\mathrm{al}^{6}$, pero difieren respecto a la sensibilidad de las plaquetas frente a la acción de E2. Estos autores observan que $5 \mathrm{uM}$ de E2 inhibe en aproximadamente 50\% (EC50) la agregación plaquetaria inducida por $1 \mathrm{ug} / \mathrm{ml}$ de colágeno. En este estudio, nosotros encontramos que $0,1 \mu \mathrm{M}$ de $\mathrm{E} 2$ inhibe en aproximadamente $80 \%$ la agregación plaquetaria inducida por $1 \mathrm{ug} /$ $\mathrm{ml}$ de colágeno. En el estudio de Wu et al, se utilizó plaquetas provenientes tanto de hombres (20-25 años de edad) como de mujeres (20-24 años de edad), en cambio, en este trabajo nosotros usamos plaquetas de mujeres postmenopáusicas (50-73 años de edad). Por otro lado, Moro et $\mathrm{al}^{8}$, usando plaquetas lavadas provenientes de hombres (2040 años de edad) observaron que $100 \mathrm{nM}$ de E2 potencia la agregación plaquetaria estimulada por trombina. Lo anterior demuestra la importancia que tiene la edad y el sexo de los donantes de sangre cuando se analiza el efecto de E2 en la agregación de plaquetas estimuladas por agentes fisiológicos.
El efecto anti-estrógenico del 27HC ha sido observado en diversos tejidos del sistema cardiovascular y óseo, donde se ha demostrado que el 27HC modula la actividad de los ERs ${ }^{5}$. Sin embargo, el 27HC presenta efectos pro-estrogénicos en hepatomas, células de cáncer al colon y líneas celulares de cáncer de mama con $\mathrm{ER}+{ }^{9}$. En el presente estudio, el 27HC actúa como un anti-estrógeno, inhibiendo el efecto anticoagulante gatillado por E2 en plaquetas activadas por colágeno. El efecto antiplaquetario de $5 \mu \mathrm{M}$ de estradiol fue drásticamente inhibido por $5 \mu \mathrm{M}$ de $27 \mathrm{HC}$. Este valor de $27 \mathrm{HC}$ puede ser considerado farmacológico, ya que en mujeres postmenopáusicas los valores observados en el suero son alrededor de $0,3 \mu \mathrm{M}$ $(0,12-0,45 \mu \mathrm{M})$. Sin embargo, las evidencias actuales parecen indicar que el $27 \mathrm{HC}$ fisiológicamente relevante sería producido localmente, así la concentración intracelular sería mayor ${ }^{10}$. En pacientes menopaúsicas con cáncer de mama $\mathrm{ER}+$, el contenido $27 \mathrm{HC}$ en el tejido normal de mama se incrementa ( 2 a 3 veces) en comparación a la de los controles sin cáncer, y el contenido de $27 \mathrm{HC}$ en el tumor es aun más elevado ( 5 a 6 veces $)^{10}$. Por otro lado, los niveles de $27 \mathrm{HC}$ en las lesiones ateroscleróticas aumentan con la severidad de la lesión y con la abundancia de macrófagos ${ }^{11}$. La propiedad del 27HC de actuar como agonista de estrógenos en algunos tejidos y como antagonista estrogénico en otros, ha llevado a considerar a este oxiesterol como un modulador selectivo del receptor de estrógenos (SERM) endógeno ${ }^{8}$.

La edad puede contribuir a modificar el metabolismo de lípidos en la menopausia, sin embargo, la menopausia per se tiene una fuerte correlación ${ }^{12}$. Diversos estudios apoyan la idea general que la menopausia natural o quirúrgica, está asociado con un perfil de lípidos no deseado. Específicamente, se ha observado una reducción del colesterol-HDL y un aumento en el colesterol-LDL, además de mayores niveles de colesterol total, fibrinógeno y lipoproteína(a). Asimismo, la menopausia está asociada con un incremento de la grasa subcutánea abdominal (Gsa) y de grasa visceral (Gv), donde el aumento de Gsa está asociado a la edad y el aumento de la adiposidad visceral está asociado a la disminución del estradiol circulante, dado el efecto que los estrógenos tienen en la actividad de la lipoproteína lipasa (a) y la lipolisis ${ }^{13}$. Si bien los estudios muestran que no hay un efecto significativo de la obesidad 
en el momento de la menopausia, las evidencias demuestran que la obesidad exacerba los síntomas del sistema vasomotor ${ }^{14}$. Además de las alteraciones hormonales y dislipidemias que se observan en la postmenopausia, durante este período los niveles séricos del $27 \mathrm{HC}$ están elevados, y este aumento estaría relacionado a la caída de los estrógenos, los cuales normalmente y vía la interacción con $\mathrm{ER} \alpha$, promueven la metabolización del 27HC a compuestos metabólicamente inactivos. La abundancia de $27 \mathrm{HC}$ es también previsible en las casos de hipercolesterolemia, situación que, como hemos analizado anteriormente, es común en la postmenopausia.

En conclusión, el hallazgo más importante de este estudio en plaquetas de mujeres postmenopáusicas es demostrar por primera vez a nivel experimental el efecto del 27-hidroxicolesterol sobre plaquetas humanas y su antagonismo estrogénico en la agregación plaquetaria por colágeno, hemos observado que el $27 \mathrm{HC}$ actúa como un anti-estrógeno, inhibiendo el efecto anticoagulante ejercido por E2. Nuestros resultados no sólo apoyan la teoría de considerar al 27HC como un SERM natural, sino también plantean la hipótesis que la hipercolesterolemia, a través de la producción de oxiesteroles, sea una importante ruta de factor de riesgo asociado a la agregación plaquetaria y su impacto en las enfermedades cardiovasculares en la mujer postmenopáusica.

Agradecimientos: Investigación financiada por Vicerrectoría ID, Proyecto Enlace ENL007/14 Universidad de Chile.

\section{Referencias}

1. Mendelsohn ME, Karas RH. Molecular and cellular basis of cardiovascular gender differences. Science 2005; 5728 : 1583-7.

2. Leitman DC, Paruthiyil S, Vivar OI, Saunier EF, Herber $\mathrm{CB}$, Cohen L, et al. Regulation of specific genes and biological responses by estrogen receptors subtype agonist. Curr Opin Pharmac 2010; 10: 629-36.
3. Lonard DM, O'Malley BW. Nuclear receptor coregulators: modulators of pathology and therapeutic target. Nat Rev Endocrinol 2012; 8: 598-604.

4. Poli G, Biasi F, Leonarduzzi G. Oxysterols in the pathogenesis of major chronic diseases. Redox Biol 2013; 1: 125-30.

5. Umetani M, Domoto H, Gormley AK, Yuhanna IS, Cummins CL, Javitt NB, et al. 27-Hydroxycholesterol is an endogenous SERM that inhibits the cardiovascular effects of estrogen. Nature Med 2007; 13: 1185-92.

6. Wu GJ, Lee JJ, Chou DS, Jayakumar T, Hsiao G, Chen $\mathrm{WF}$, et al. Inhibitory signaling of 17 beta-estradiol in platelet activation: the pivotal role of cyclic AMP-mediated nitric oxide synthase activation. Eur J Pharmacol 2010; 649: 140-9.

7. Garrido A, Muñoz Y, Sierralta W, Valladares L. Metabolism of dehydroepiandrosterone sulfate and estrone-sulfate by human platelets. Physiol Res 2012; 61: 381-8.

8. Moro L, Reineri S, Piranda D, Pietrapiana D, Lova P, Bertoni A, et al. Nongenomic effects of 17 (beta)-estradiol in human platelets potentiation of thrombin-induced aggregation through estrogen receptor (beta) and Src kinase. Blood 2005; 105: 115-21.

9. Cruz P, Torres C, Ramírez ME, Epunan MJ, Valladares LE, Sierralta WD. Proliferation of human mammary cancer cells exposed to 27-hydroxycholesterol. Exp Ther Med 2010; 1: 531-6.

10. Wu Q, Ishikawa T, Sirianni R, Tang H, McDonald JG, Yuhanna IS, et al. 27-Hydroxycholesterol promotes cell-autonomous, ER-positive breast cancer growth. Cell Rep 2013; 5: 637-45.

11. Al-Safi ZA, Polotsky AJ. Obesity and menopause. Best Pract Res Clin Obstet Gynaecol 2015; 29: 548-53.

12. Stevenson JC, Crook D, Godsland IF. Influence of age and menopause on serum lipids and lipoproteins in healthy women. Atherosclerosis 1993; 98: 83-90.

13. Gold EB, Bromberger J, Crawford S, Samuels S, Greendale GA, Harlow SD, et al. Factors associated with age at natural menopause in a multiethnic sample of midlife women. Am J Epidemiol 2001; 153: 865-74.

14. Tang W, Eggertsen G, Chiang JY, Norlin M. Estrogen-mediated regulation of CYP7B1: a possible role for controlling DHEA levels in human tissues. J Steroid Biochem Mol Biol 2008; 112: 63-73. 